\title{
Dynamic Interactions between a Buoyant Reacting Plume and Evaporating Droplets
}

\author{
JUN XIA ${ }^{1}$, KAI H. LUO ${ }^{1}$ and SURESH KUMAR ${ }^{2 \dagger}$ \\ 1. Energy Technology Research Group, School of Engineering Sciences, University of \\ Southampton, Highfield, Southampton SO17 1BJ, UK \\ 2. BRE Global Limited, Garston, Watford WD25 9XX, UK; KumarS@bre.co.uk
}

\section{ABSTRACT}

This paper reports a fundamental study of dynamic interactions between a buoyant reacting plume and evaporating droplets using large-eddy simulation (LES). An idealised prototype configuration is set up to numerically mimic a sprinkler nozzle placed right above the fire source, dispensing evaporating droplets of various properties. The gas phase of the reacting plume is described in the Eulerian frame while the discrete droplet phase is treated in the Lagrangian frame, with strong two-way coupling between the two phases through mass, momentum and energy exchange. Finite-rate chemistry is included and modelled by a scale similarity subgrid-scale model. The LES has six dynamically determined model constants, which minimizes constant tuning. A parametric study has been conducted by varying the initial Stokes number $\left(S t_{0}\right)$ or non-dimensional droplet size, mass loading ratio $\left(M L R_{0}\right)$ and droplet velocity magnitude $\left(\left|\mathbf{v}_{\mathrm{d} 0}\right|\right)$, independently. Droplets of three initial sizes $(780 \mu \mathrm{m}, 390 \mu \mathrm{m}$ and $195 \mu \mathrm{m})$ have distinctively different dynamic interactions with the buoyant reacting plume. Increasing $M L R_{0}$ progressively from 0 to 9 increases the droplet effects, and in the case of the largest droplets used, the reaction is completely suppressed and the plume structure destroyed. Increasing $\left|\mathbf{v}_{\mathrm{d} 0}\right|$ has mixed effects on the droplets' fire suppression capacity. Detailed analysis of the budget equation for a non-dimensional gas temperature reveals roles played by the droplet-related terms in combustion suppression. The only "cooling" effect on gas temperature comes from the convective heat transfer between the phases, which drives droplet vaporisation, while there are three mechanisms (mechanical work done by droplet drag force, part of internal energy transfer into the gas phase due to evaporation and kinetic energy interactions between the phases) contributing to "warming" effects. On the whole, evaporating droplets in all cases studied result in significant reduction in reaction rate and gas temperature especially the peak values.

KEYWORDS: buoyant diffusion flames, fire suppression, water spray, modelling, LES

\section{NOMENCLATURE LISTING}

\begin{tabular}{|c|c|c|c|}
\hline$D_{\mathrm{d}}$ & droplet diameter & $T_{\mathrm{a}}$ & time-averaging period for statistics \\
\hline$D a$ & Damköhler number & $\Delta t$ & time step \\
\hline$d_{\mathrm{v} 50}$ & volumetric median droplet diameter & $t$ & time \\
\hline$e$ & internal energy & $\mathbf{u}$ & gas velocity vector \\
\hline$e^{\prime}$ & sensible energy & $V$ & LES filtering volume \\
\hline $\mathbf{F}_{\text {drag }}$ & $\begin{array}{l}\text { vector of drag force exerted on } \\
\text { droplets }\end{array}$ & $\mathbf{v}$ & droplet velocity vector \\
\hline$F r$ & Froude number & $Z e$ & Zel'dovich number \\
\hline$h_{\mathrm{fg}}$ & latent heat of vaporisation & Greek & \\
\hline$L_{\text {nozzle }}$ & sprinkler nozzle width & $\gamma$ & ratio of specific heats \\
\hline$L_{x}, L_{y}, L_{z}$ & computational domain size & $\theta_{0}$ & spray discharge angle \\
\hline$m$ & mass & $\theta_{\mathrm{d} 0}$ & $\begin{array}{l}\text { initial discharge angle of a water } \\
\text { droplet }\end{array}$ \\
\hline$M a$ & Mach number & $\rho$ & density \\
\hline$V F L$ & $\begin{array}{l}\text { volumetric flow rate of the sprinkler } \\
\text { nozzle }\end{array}$ & $\sigma_{i j}$ & grid-scale shear stress tensor \\
\hline$M L R$ & $\begin{array}{l}\text { mass loading ratio, defined as the } \\
\text { mass flow rate of liquid droplets at the } \\
\text { sprinkler nozzle to that of fuel gas at } \\
\text { the fire source }\end{array}$ & $\tau_{i j}$ & $\begin{array}{l}\text { subgrid-scale shear stress tensor } \\
\left(\tau_{i j}=\widetilde{u_{\mathrm{g}, i} u_{\mathrm{g}, j}}-\widetilde{u_{\mathrm{g}, i} u_{\mathrm{g}, j}}\right)\end{array}$ \\
\hline$N_{\mathrm{d}}$ & total droplet number in the domain & $|\omega|$ & vorticity magnitude \\
\hline
\end{tabular}




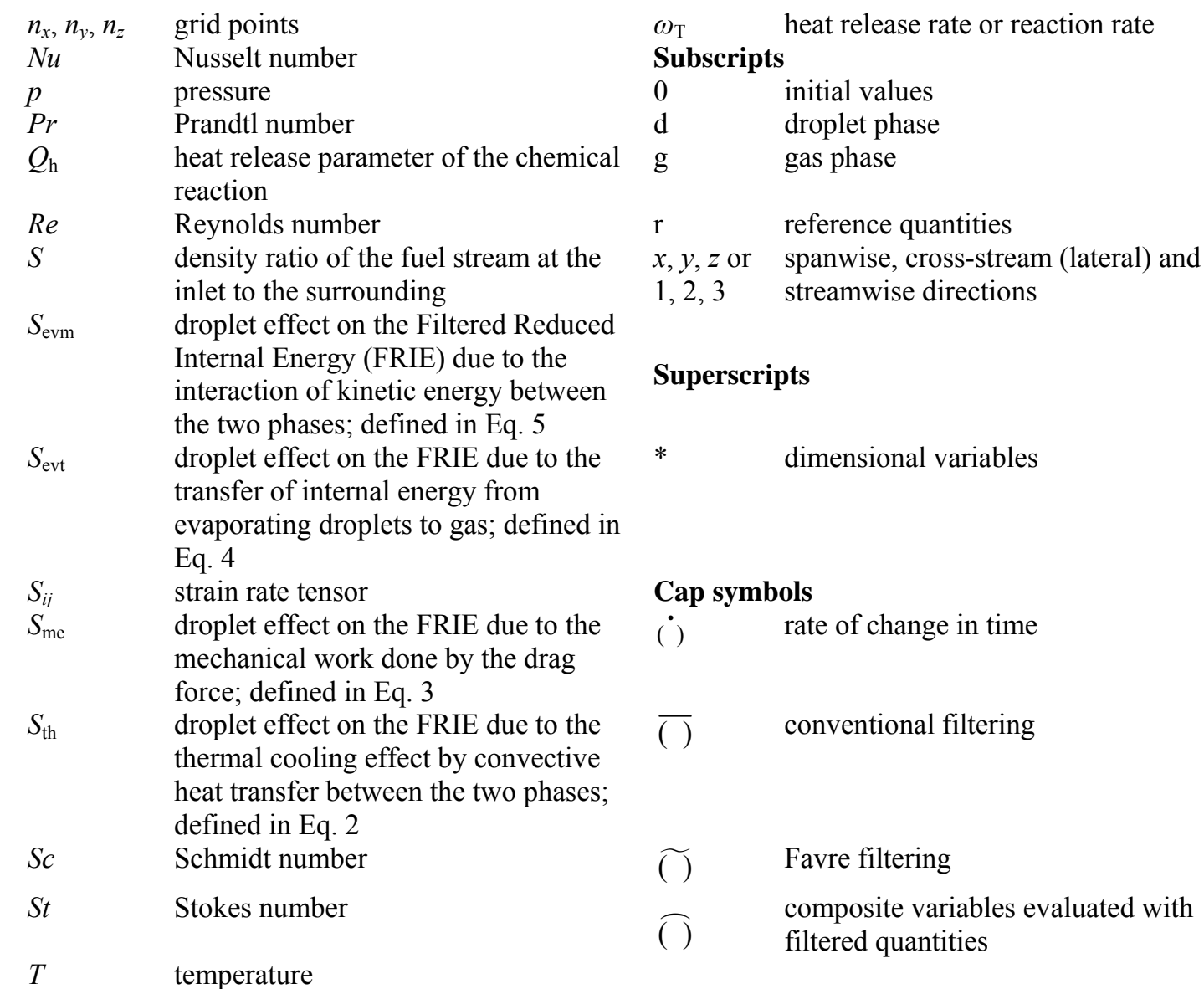

\section{INTRODUCTION}

Fire control and mitigation is as important today as it has been for centuries. Over the past hundred years, water spray sprinkler systems have been developed to the point where they provide a highly effective means of controlling, suppressing and extinguishing fire in buildings. Since the ban of Halon gas based systems due to environmental concern, water-based fire suppression technology has received renewed attention [1]. Better understanding of the fundamental phenomena involved is crucial for optimising the design, which has motivated a number of recent studies in the field [2-10]. Naturally, experiments have advantages in being able to study fire suppression by water spray or mist under realistic conditions. Since the early work of McCaffrey [2,3], there have been increasing number and more detailed experimental investigations [4-10], covering a wide range of configurations and physical parameters. On the other hand, experiments are expensive and in most cases difficult to provide detailed spatially and temporally resolved data.

Numerical simulations, in contrast, are able to provide temporally resolved, full-field data of multiple quantities, providing a valuable tool for fundamental research and a cost-effective design tool. However, numerical techniques have not been fully developed to simulate fire suppression systems under realistic conditions, although considerable progress has been made in the last decade. So far, all numerical simulations have involved considerable simplifications. Prasad et al. simulated two-dimensional (2D) liquid pool fire [11] and co-flow diffusion flame [12] under the influence of water mist. Laminar flames interacting with water droplets in a counterflow configuration were studied by Lentati and Chelliah [13]. Using the RANS (Reynolds-Averaged Navier-Stokes) approach, Kumar et al. [14] and Holborn et al. [15] employed the JASMINE-SPARTA model to examine the effect of sprinklers on fire gases; Nam [16, 17] investigated the interaction between a fire plume and a sprinkler spray, while Yoon et al. [18] studied suppression of large-scale compartment fires using water spray. The RANS approach, however, has known weaknesses for highly unsteady and turbulent phenomena such as the fire suppression scenario. A more 
powerful simulation method is Large-Eddy Simulation (LES), which has gained popularity in fire research in recent years [19], as computing power for such an approach has become available. The Fire Dynamics Simulator (FDS) developed by NIST has shown the capability of LES in various fire scenarios. LES has been recently employed by the present authors to investigate the dynamics of turbulent reacting jets diluted with water droplets under various initial droplet sizes and injection densities [20].

The objective of the present paper is to apply the developed LES methodology [20] to a fundamental study of dynamic interactions between water spray from a virtual slit source interacting with an upward buoyant reacting plume. The configuration is different from most used in previous numerical studies and has a prototype value. The objective is to investigate the mechanisms of multilateral interactions among turbulent flow, droplet dynamics and chemical reactions through a parametric study.

\section{MATHEMATICAL FORMULATIONS AND NUMERICAL METHODS}

A hybrid Eulerian-Lagrangian approach is used for the gas-liquid two-phase reacting system. The complete set of nondimensionalized governing equations includes the three-dimensional time-dependent NavierStokes equations, the transport equations for the fuel gas, oxidizer gas, water vapour, and the evaporating droplets [20]. The governing equations for the gas phase are solved in the Eulerian frame while the discrete phase is treated in the Lagrangian frame. The two-way coupling between the continuum and the discrete phases is included in the source terms, which account for mass, momentum and energy exchange [20].

In this paper, all quantities for both phases are normalised using the usual reference values as follows: The reference density $\rho_{\mathrm{r}}^{*}$, length $l_{\mathrm{r}}^{*}$, velocity $u_{\mathrm{r}}^{*}$, temperature $T_{\mathrm{r}}^{*}$ and viscosity $\mu_{\mathrm{r}}^{*}$ are respectively the ambient air density, fire source width, inlet velocity, environmental air temperature and viscosity; Besides, the reference time $t_{\mathrm{r}}^{*}=l_{\mathrm{r}}^{*} / u_{\mathrm{r}}^{*}$, reference pressure $p_{\mathrm{r}}^{*}=\rho_{\mathrm{r}}^{*} u_{\mathrm{r}}^{* 2}$, reference internal energy and reference enthalpy $e_{\mathrm{r}}^{*}=h_{\mathrm{r}}^{*}=u_{\mathrm{r}}^{* 2}$. The superscript “*” denotes dimensional quantities. Specifically, $l_{\mathrm{r}}^{*}=3.34 \mathrm{~cm}$ and $u_{\mathrm{r}}^{*}=1.81 \mathrm{~m} / \mathrm{s}$ in this paper, as the ambient air properties are set at $1 \mathrm{~atm}$ and $20^{\circ} \mathrm{C}$. To facilitate the understanding, both dimensionless and the correspondingly dimensional values of the coordinates have been shown in all the figures presented in this paper. The spanwise, cross-stream and streamwise (vertical) directions are denoted $x, y$ and $z$, respectively. The corresponding indices in the tensor notation are 1, 2 and 3. The symbols " ${ }^{-} ",{ }^{\sim} "$ and $"{ }^{-}$designate the normal filtering, Favre filtering and composite variables evaluated with filtered quantities, respectively.

To simulate a realistic fire suppression scenario using LES is still prohibitively expensive. In this study, we limit our aim to improving fundamental understanding of key phenomena in such a scenario in an idealized prototype. Figures 1 and 2 illustrate the computational domain in which a droplet source is placed at a height of $z=38$. The shape and size of the droplet source (slit nozzle) is the same as that of the fuel jet nozzle. In reality, sprinklers have different geometric designs, operating pressures and spray angles, etc., leading to a wide variety of atomization mechanisms, initial momenta and droplet size distributions and so on. Even with one particular sprinkler design, polydisperse droplets of a wide range of diameters and momenta are delivered. While all these different droplet properties can in principle be specified in a numerical simulation, it is a formidable task and a computationally expensive exercise to attempt all scenarios. If one believes that there are common underlying mechanisms behind the different scenarios, then a fundamental study should focus on a simplified prototype problem. In this study, monodisperse water droplets are initially discharged from the sprinkler nozzle using a two-dimensional uniform random distribution. The initial discharge angle of a water droplet, $\theta_{\mathrm{d} 0}$, is set by $\theta_{\mathrm{d} 0}=270^{\circ}+\theta_{0}\left(y_{\mathrm{d} 0}-L_{y} / 2\right) / L_{\text {nozzle }}$, where $\theta_{0}$ is the spray angle of a fire sprinkler; $y_{\mathrm{d} 0}$ is the initial random lateral coordinate of the droplet; $L_{\text {nozzle }}$ is the lateral width of the slit sprinkler nozzle $\left(L_{\text {nozzle }}=1\right.$ and $L_{\text {nozzle }}^{*}=3.34 \mathrm{~cm}$ in this study). All the water droplets enter the computational domain with a uniform velocity magnitude $\left|\mathbf{v}_{\mathrm{d} o}\right|$. The initial lateral and streamwise velocity for every droplet are then decided by $v_{\mathrm{d} 0}=\left|\mathbf{v}_{\mathrm{d} 0}\right| \sin \theta_{\mathrm{d} 0}, w_{\mathrm{d} 0}=\left|\mathbf{v}_{\mathrm{d} 0}\right| \cos \theta_{\mathrm{d} 0}$. The initial spanwise velocity of the droplet, $u_{\mathrm{d} 0}$, is set to 0 . Similar initial conditions for water spray droplets were used in [16] by Nam. The modeled fire sprinkler is activated at $t=100$, when the fire plume, accelerated by the strong buoyancy force $(F r=10)$, has been established in the computational domain. 
Equally, the physical processes involved have to be simplified. The classical rapid mixing model [21] is used to describe droplets evaporation. A one-step irreversible reaction with Arrhenius-type reaction rate [22] is employed, which with properly calibrated parameters is capable of representing the main features of hydrocarbon combustion [23]. The chosen values for the Damköhler, $D a$ and Zel'dovich number, Ze, the heat release parameter, $Q_{\mathrm{h}}$, respectively, are shown in Table 1 . As radiative heat transfer is not included, these combustion parameters are selected carefully to compensate for radiative heat loss, so that the peak temperature is in the range of a typical small fire. To model the filtered reaction, a scale similarity model [24] is employed. Reduced gravitational effects are included with the Froude number $(\mathrm{Fr})$ equal to 10. Transport and thermochemical properties are also simplified, for example, with the Prandtl number $\operatorname{Pr}$ and Schmidt number $S c$ set to a constant of 0.7 .

High-order numerical schemes (up to $6^{\text {th }}$-order) and compatible numerical boundary conditions are employed. For the gas phase, the NSCBC (Navier-Stokes Characteristic Boundary Conditions) [25] is deployed at the inflow and lateral boundaries. At the outflow boundary, the NRBC (Non-Reflecting Boundary Conditions) [26] is applied at the end of the physical domain $z=40$. To minimize the numerical wave reflections at the open outflow boundary, a sponge layer [27] ranging from $z=40$ to $z=42.785$ is employed. The spanwise direction is periodic for both phases. Any droplets leaving from the inflow, lateral and outflow boundaries are assumed not to enter the computational domain again and deleted from the droplet list. The gas phase equations are solved by an LES, which uses dynamic eddy-viscosity and eddydiffusivity subgrid-scale (SGS) models. Six SGS model constants in the momentum, energy and species equations are determined by the Germano dynamic procedure [28]. The gas properties at the droplet locations are obtained by a $4^{\text {th }}$-order Lagrangian interpolation scheme. Time advancement is achieved by a $3^{\text {rd }}$-order Runge-Kutta method with the CFL number set to 1.5 . The ratio of the time step $\Delta t$ to the characteristic droplet time scale, $\Delta t / S t$ in the non-dimensional context, for every droplet is monitored throughout the simulation period to ensure the temporal resolution for both phases.

Table 1 presents the key simulation and physical parameters. Both dimensionless and dimensional values have been listed for the initial droplet parameters for clarity. The Stokes number $S t$ is defined as the ratio of a characteristic droplet responsive time to a characteristic flow time, $S t=\tau_{\mathrm{d}}^{*} / \tau_{\mathrm{g}}^{*}=R e \cdot \rho_{\mathrm{d}} D_{\mathrm{d}}^{2} /(18 \mu)$, and denotes the non-dimensional droplet size as $S t \sim D_{\mathrm{d}}^{2}$. The mass loading ratio $M L R_{0}$ is that of the liquid mass flow rate at the sprinkler nozzle to the gas flow rate at the inlet. $V F L^{*}$, whose unit is $L / \mathrm{min}$, designates the volumetric flow rate of the sprinkler nozzle. Other initial parameters include: the spray discharge angle $\theta_{0}$, initial Reynolds number $R e$, latent heat of vaporization of water $h_{\mathrm{fg}}$, the density ratio of the fuel stream to the surrounding $S$, the computational domain size and grid. The equivalent dimensional domain size is $L_{x}^{*} \times L_{y}^{*} \times L_{z}^{*}=26.7 \times 106.2 \times 142.9 \mathrm{~cm}^{3}$. The initial droplet diameter for $S t_{0}=100$ is $D_{\mathrm{d} 0}^{*} \approx 780 \mu \mathrm{m}$, and the dimensional initial droplet velocity magnitude for $\left|\mathbf{v}_{\mathrm{d} 0}\right|=2$ is $\left|\mathbf{v}_{\mathrm{d} 0}^{*}\right| \approx 3.62 \mathrm{~m} / \mathrm{s}$, both of which have been decided with reference to the values for standard spray fire sprinklers used for suppression of small-scale fires $[8,9]$. The droplet number in the domain $N_{\mathrm{d}}$ varies. For example, $N_{\mathrm{d}}$ is close to half a million for Case $\mathrm{D}$ at time $t=150$ shown in Fig. 2.

Table 1. Simulation parameters and cases. $R e=4000, S=0.76, F r=10, D a=80, Z e=8.5, Q_{\mathrm{h}}=250, h_{\mathrm{fg}}=250$, $\theta_{0}=50^{\circ}$. The computational domain size $L_{x} \times L_{y} \times L_{z}=8 \times 31.8 \times 42.785$, and the grid $n_{x} \times n_{y} \times n_{z}=41 \times 160 \times 200$.

\begin{tabular}{c|c|c||c|c||c|c}
\hline \hline Cases & $S t_{0}$ & $D_{\mathrm{d} 0}^{*}(\mu m)$ & $M L R_{0}$ & $\begin{array}{c}V F L^{*} \\
(L / m i n)\end{array}$ & $\left|\mathbf{v}_{\mathrm{d} 0}\right|$ & $\left|\mathbf{v}_{\mathrm{d} 0}^{*}\right|(\mathrm{m} / \mathrm{s})$ \\
\hline $\mathrm{A}$ & - & - & 0 & 0 & - & - \\
\hline $\mathrm{B}$ & 100 & 780 & 3 & 2.74 & 2 & 3.62 \\
\hline $\mathrm{C}$ & 25 & 390 & 3 & 2.74 & 2 & 3.62 \\
\hline $\mathrm{D}$ & 6.25 & 195 & 3 & 2.74 & 2 & 3.62 \\
\hline $\mathrm{E}$ & 100 & 780 & 6 & 5.48 & 2 & 3.62 \\
\hline $\mathrm{F}$ & 100 & 780 & 9 & 8.22 & 2 & 3.62 \\
\hline $\mathrm{G}$ & 100 & 780 & 3 & 2.74 & 3 & 5.43 \\
\hline $\mathrm{H}$ & 100 & 780 & 3 & 2.74 & 4 & 7.24 \\
\hline \hline
\end{tabular}




\section{RESULTS AND DISCUSSION}

\section{Effects of Initial Droplet Size}

A key consideration of a water-based fire suppression system is the initial size of the droplets disseminated at the nozzle [1, 17]. Competing requirements exist among droplet number density, rate of evaporation, penetration capacity and so on for the same amount of water released. In this study, we compare the performance of droplets of three initial diameters: $780 \mu \mathrm{m}, 390 \mu \mathrm{m}$ and $195 \mu \mathrm{m}$, corresponding to $S t_{0}=100,25$ and 6.25 , respectively. The first is within the typical range of the volumetric median droplet diameter, $d_{\mathrm{v} 50}$, for small fire suppression systems [8,9], and the third is below 200 $\mu \mathrm{m}$ and can be considered as mist. Shown in Fig. 1 is a typical reacting plume structure for Case B $\left(S t_{0}=100\right)$. The near field shows the characteristic large coherent structures in a buoyant reacting plume. A transition occurs following the rapid growth and interaction of shear-layer instabilities and buoyancy-driven instabilities. The field downstream exhibits typical turbulent reacting flow behaviour due to buoyancy acceleration even though the inlet Reynolds number is moderate.

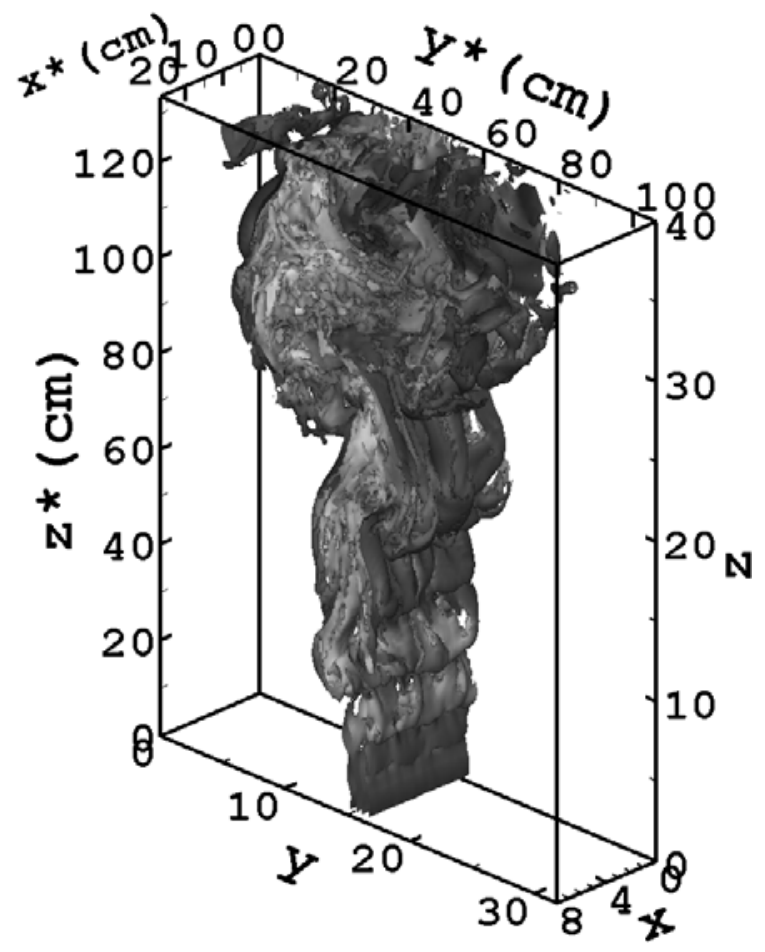

Fig. 1. Iso-surfaces of the vorticity magnitude $|\omega|=1$ at $t=180$ for Case $\mathrm{B}$, showing the reacting plume structure under insignificant suppression by droplets. The colours indicate local gas temperature, with white showing high temperature.

In Fig. 2, the droplet distributions are shown for Cases B, C and D, exhibiting three distinct patterns. The large droplets of $S t_{0}=100$ are able to penetrate the whole reacting plume and reach the fire base, forming an ideal pattern in order to control the flame growth and spread. The medium droplets of $S t_{0}=25$ are held up in the central plume region, referred to as the "interaction boundary" [8], due to strong buoyancy-driven flow acceleration there. In the peripheral regions, however, droplets can overcome the upward flow momentum and reach deeper towards the outer region of the fire base. With the smallest droplets of $S t_{0}=6.25$, there is also an "interaction boundary" in the central region, but no droplets are found below $z=10$, due to relatively stronger rising plume. The penetration capacity of mist droplets may be improved by increasing the operating pressure of the sprinkler nozzle, and different interaction regimes may be observed between mist and a reacting plume. This topic is however beyond the scope of the present study. Consequently, the following sections will focus on the spray droplets of $S t_{0}=100$ only. 

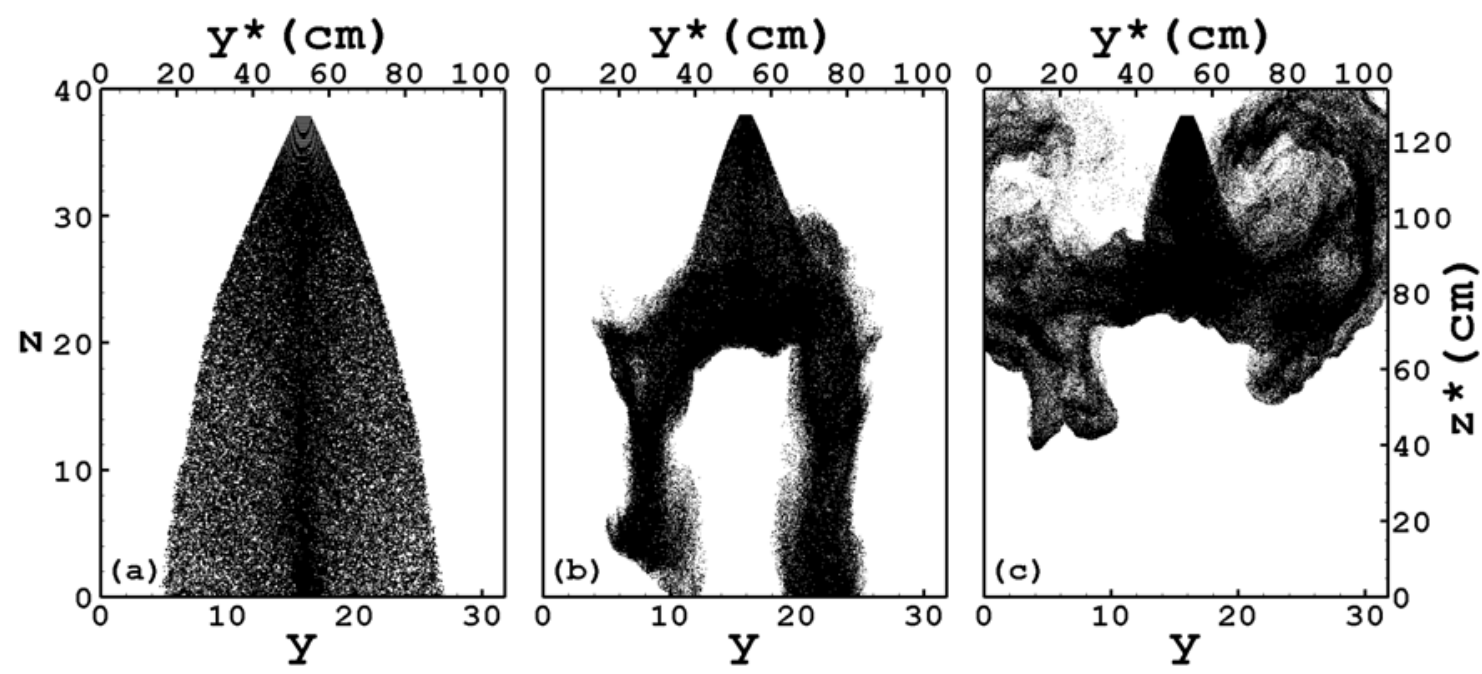

Fig. 2. The instantaneous droplet distribution at $t=150$

for (a) Case B, (b) Case C and (c) Case D, showing three distinct regimes.

\section{Effects of Initial Droplet Momentum}

The initial droplet momentum of a sprinkler is an important parameter that in practice is determined by the operating pressure of the sprinkler [29]. Numerically, the droplet momentum effects can be studied by varying the mass loading ratio $\left(M L R_{0}\right)$ or initial droplet velocity magnitude $\left(\left|\mathbf{v}_{\mathrm{d} 0}\right|\right)$. Cases $\mathrm{B}, \mathrm{E}$ and $\mathrm{F}$ examine the effects of increasing $M L R_{0}$ while keeping $\left|\mathbf{v}_{\mathrm{d} 0}\right|$ constant. At time $t=180$, droplets in all cases have fully penetrated the reacting plume and reached the fire base. With increasing $M L R_{0}$, the reaction and plume temperature are suppressed to an ever greater extent due to heat exchange between the droplets and the plume. Figure 3 shows the temperature field of Case F in comparison with that of Case A (without droplets). As the source of droplets is directly over the fire base, the plume central region bears the brunt of the droplet effects, leading to severe reduction in temperature there. However, the reacting plume dynamically adjusts itself by expanding in the lateral direction and high-temperature zones (with reaction) manage to survive in the peripheral regions. Due to the large $M L R_{0}$ in Case $\mathrm{F}$, the flow field and the plume structure are significantly changed due to momentum exchange between the gas and droplet phases. In fact, there is a large region of flow reversal (see Fig. 4) directly under the droplet source, as the drag effects are strong with high droplet number density. The results of Cases B and $\mathrm{E}$ are qualitatively similar but quantitatively different. In Case B, for example, the plume structure is only slightly modified rather than distorted. Droplet effects on flow are further illustrated in Fig. 4, where time history of the streamwise velocity $w$ at a downstream point along the central line $[x, y, z]=[4,15.8,24.08]$ is shown for various cases. Compared with the no-droplet Case A, the velocity $w$ of Case B shows reduced magnitude and delayed phase but no flow reversal. For Cases $\mathrm{E}$ and $\mathrm{F}, w$ becomes negative at $t \approx 165$ and 140 , respectively, following droplet release from $t=100$.

The effects of changing the initial droplet velocity can also be gauged by looking at the records of Cases $\mathrm{G}$ and $\mathrm{H}$ in Fig. 4. With a higher $\mid \mathbf{v}_{\mathrm{do}}$, droplets in Case $\mathrm{G}$ and $\mathrm{H}$ have 1.5 and 2 times the momentum of those in Case B, but their effects on $w$ are not much greater than in Case B. More strikingly, droplets in Cases $\mathrm{H}$ and $\mathrm{E}$ have the same momentum, but Case $\mathrm{E}$ has significant flow reversal while Case $\mathrm{H}$ does not. One reason for the apparent ineffectiveness (in fire suppression) of increasing $\left|\mathbf{v}_{\mathrm{d} 0}\right|$ is the shorter time scale for momentum and heat interaction between the droplets and the reacting plume. Moreover, the shorter residence time of droplets means fewer droplets inside the computational domain and consequently smaller effects on the reacting plume.

It should be emphasized that the above observations apply to the present prototype problem. The effects of droplets in a realistic sprinkler may be quantitatively different. 

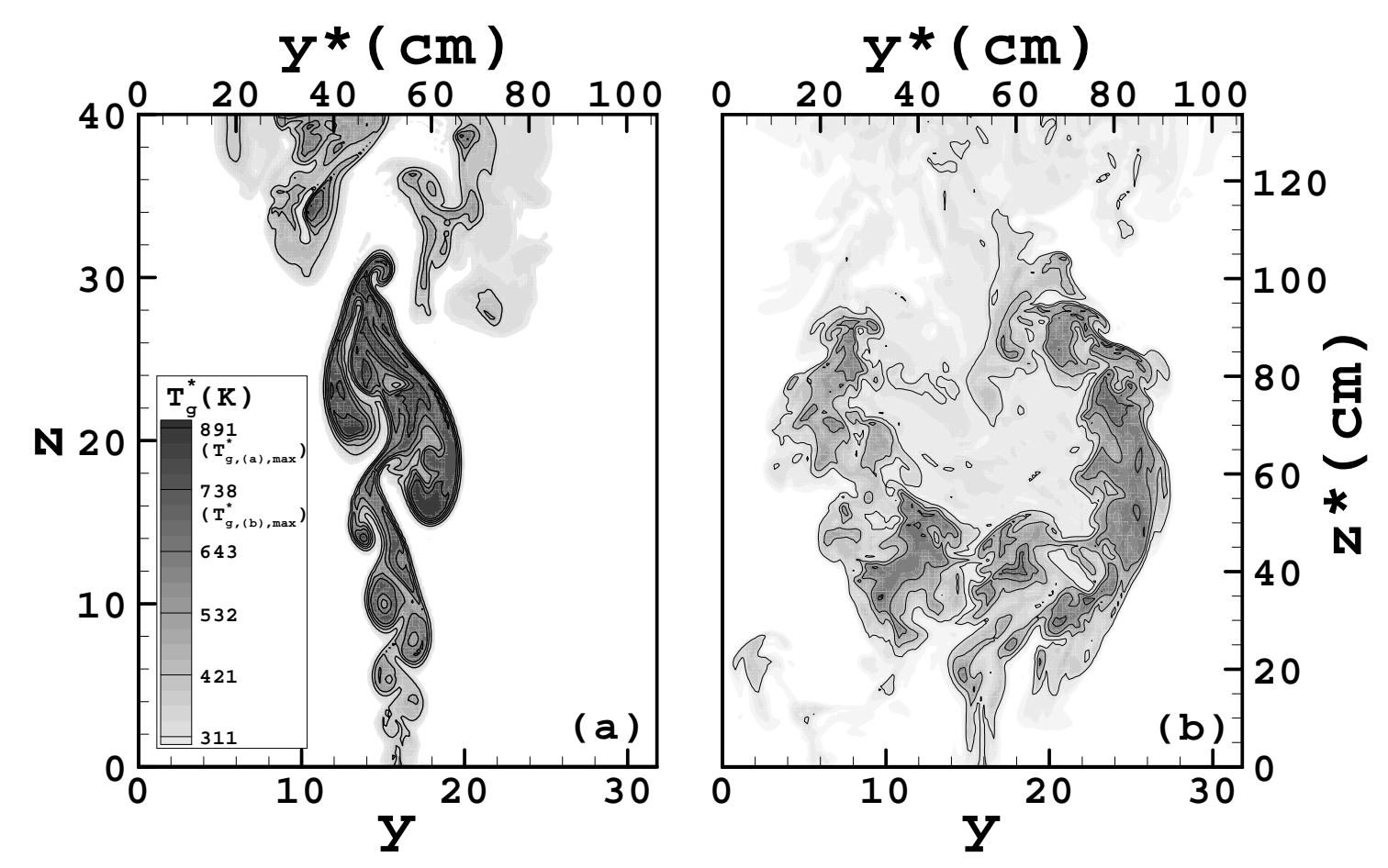

Fig. 3. The temperature field in the central plane in the spanwise direction at $t=180$

for (a) Case A and (b) Case F. The same ranges of flood and line contour levels are used for (a) and (b).

The highest local temperature is $891 \mathrm{~K}$ in (a) and $738 \mathrm{~K}$ in (b), respectively.

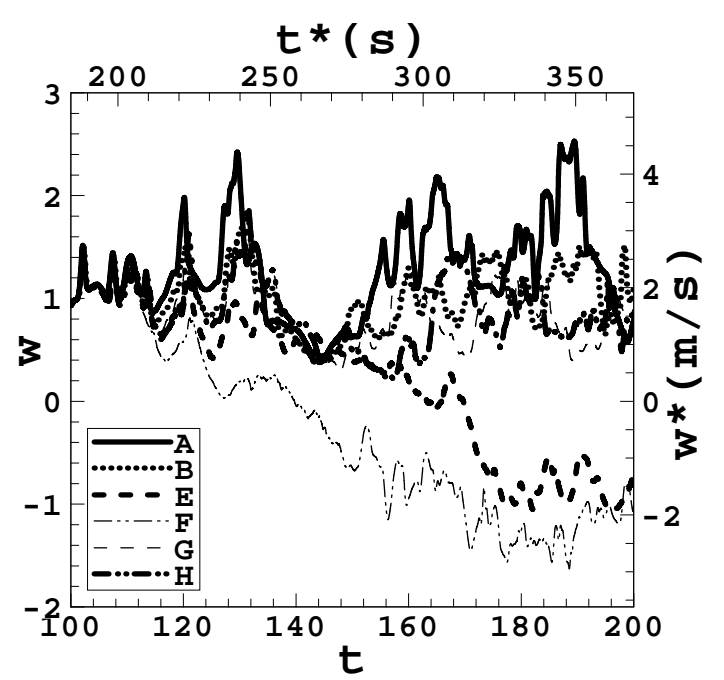

Fig. 4. Time records of the streamwise velocity at a downstream point on the plume axis of the central spanwise plane $[x, y, z]=[4,15.8,24.08]$ for six cases with and without evaporating droplets.

\section{Droplet Thermal Effects}

Thermal interactions between the gas and droplet phases involve several mechanisms including convective heat transfer, radiation, phase change and so on. Radiative heat exchange among the droplets, the reacting plume and the surrounding (especially an enclosure) would play an intricate role in the fire suppression scenario. To isolate the effects of radiation merits a separate study. In this study, radiation is not considered but convective heat transfer and droplet evaporation are included. To further understanding of the nonlinear 
thermal effects, we examine a transport equation for a non-dimensional gas temperature called the Filtered Reduced Internal Energy (FRIE) of the two-phase reacting flow, $\overline{\rho_{\mathrm{g}} e_{\mathrm{g}}^{\prime}}$, defined as $\overline{\rho_{\mathrm{g}} e_{\mathrm{g}}^{\prime}}=\overline{\rho_{\mathrm{g}}} \widetilde{\mathrm{g}} /\left\{\gamma(\gamma-1) M a^{2}\right\}$. The transport equation for FRIE can be written as

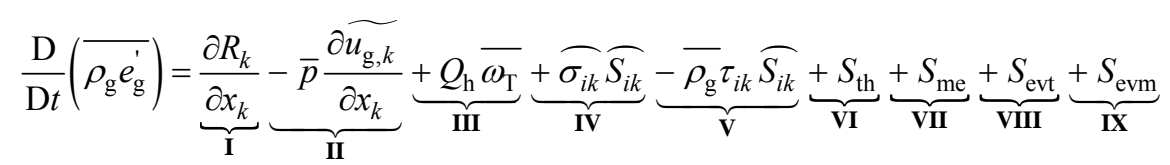

where

$$
\begin{aligned}
& S_{\mathrm{th}}=-\frac{1}{V} \sum_{k} s_{\mathrm{th}, k}=-\frac{1}{V} \sum_{k}\left[\frac{1}{3} \frac{N u_{k}}{(\gamma-1) M a^{2} P r} \frac{m_{\mathrm{d}, k}}{S t_{k}}\left(T_{\mathrm{g}, k}-T_{\mathrm{d}, k}\right)\right] \\
& S_{\mathrm{me}}=-\frac{1}{V} \sum_{k} s_{\mathrm{me}, k}=-\frac{1}{V} \sum_{k}\left[-F_{\mathrm{drag}, k, i}\left(\widetilde{u_{\mathrm{g}, i}}-v_{\mathrm{d}, k, i}\right)\right] \\
& S_{\mathrm{evt}}=-\frac{1}{V} \sum_{k} s_{\mathrm{evt}, k}=-\frac{1}{V} \sum_{k}\left[\frac{1}{(\gamma-1) M a^{2}} \dot{m}_{\mathrm{d}, k} T_{\mathrm{d}, k}\right] \\
& S_{\mathrm{evm}}=-\frac{1}{V} \sum_{k} s_{\mathrm{evm}, k}=-\frac{1}{V} \sum_{k}\left[\frac{1}{2} \dot{m}_{\mathrm{d}, k}\left(\widetilde{u_{\mathrm{g}, i}}-v_{\mathrm{d}, k, i}\right)^{2}\right]
\end{aligned}
$$

In Eqs. 1-5, the subscripts "g" and "d" denote the gas and the droplet phases, respectively. Most symbols have their usual meanings. $\widetilde{\sigma_{i k}}$ and $\overline{\rho_{\mathrm{g}}} \tau_{i k}$ are the grid-scale and subgrid-scale stress tensors, respectively. $\widetilde{S_{i k}}$ is the strain rate tensor. $\mathbf{F}_{\text {drag }}$ is the drag force exerted on droplets by the reacting flow. Term I $\left(\partial R_{k} / \partial x_{k}\right)$ represents all the redistributive terms, which are neither net sources nor sinks, and therefore are not traced. Terms II-V designate pressure dilatation, combustion heat release, grid scale dissipation rate and subgrid scale dissipation rate, respectively. Among the droplet-related terms, $S_{\mathrm{th}}$ represents thermal cooling due to convective heat transfer between the two phases; $S_{\mathrm{me}}$ the mechanical work done by the drag force; $S_{\text {evm }}$ a contribution arising from the interaction of kinetic energy between the two phases; and $S_{\text {evt }}$ a (small) portion of droplet internal energy which is transferred into the gas after evaporation. Note the rate of change of the internal energy of a single droplet $d\left(m_{\mathrm{d}}{ }^{*} c_{\mathrm{d}}^{*} T_{\mathrm{d}}^{*}\right) / d t^{*}=d m_{\mathrm{d}}^{*} / d t^{*} \cdot c_{\mathrm{d}}^{*} T_{\mathrm{d}}^{*}+m_{\mathrm{d}}^{*} c_{\mathrm{d}}^{*} \cdot d T_{\mathrm{d}}^{*} / d t^{*}=s_{\mathrm{evt}}^{*}+$ $s_{\mathrm{th}}^{*}+\dot{m}_{\mathrm{d}}^{*} h_{\mathrm{fg}}^{*}$, where $c_{\mathrm{d}}^{*}$ is the specific heat of liquid droplets and assumed to be a constant. $\dot{m}_{\mathrm{d}}^{*} h_{\mathrm{fg}}^{*}$ has been excluded from the current analysis since it directly affects the total internal energy of the gas phase $\rho_{\mathrm{g}}^{*} e_{\mathrm{g}}^{*}$, but not the reduced internal energy $\rho_{\mathrm{g}}^{*} e_{\mathrm{g}}^{* *}$ (FRIE). As shown below, $s_{\mathrm{th}}^{*}$ is a dominant sink term while $s_{\mathrm{evt}}^{*}$ is a small source term for the FRIE.

Statistics of the budget terms in Eq. 1 are shown in Fig. 5 for Cases A, B, G and H obtained by time averaging during the period $T_{\mathrm{a}}=[140,200]$ and spatial averaging over the spanwise direction. From the time when the flow data are recorded for the averaging purpose, i.e., $t=140$, droplets have covered the whole plume region from the sprinkler nozzle down to the fire source. The reacting plume then experiences approximately another two "droplet-through time" periods, correspondingly one "flow-through time" period, for data recording. Here, one "droplet-through time" period is defined as the period from the instant when droplets are discharged from the sprinkler nozzle to the instant when these droplets leave the inflow boundary, which is about 30 time units for the $S t_{0}=100$ droplets. One "flow-through time" period is the period from the instant when the reactive gases enter the computational domain from the inflow boundary to the instant when they move out of the outflow boundary due to convection and buoyancy, which is about 60 time units for the pure reacting plume Case A. The spatial ensemble averaging over the spanwise direction is performed finally. No such statistics are presented for Cases E and F, as these cases are highly unsteady and time averaging is not appropriate. The dimensional counterparts of all the right-hand-side 
terms in Eq. 1 have a unit $w / m^{3}$ as in Fig. 5. A prominent phenomenon is that the convective heat transfer term VI $\left(S_{\mathrm{th}}\right)$ is the only term that has "cooling" effects on the gas, while terms VII $\left(S_{\mathrm{me}}\right)$, VIII $\left(S_{\mathrm{evt}}\right)$ and IX $\left(S_{\text {evm }}\right)$ all have "warming" effects. The kinetic energy interaction term IX $\left(S_{\text {evm }}\right)$ is the smallest contribution among all the droplet-related terms. The reason why VIII $\left(S_{\text {evt }}\right)$ has a "warming" effect is that evaporation transforms liquid into gas and represents an energy gain for the gas phase. However, its magnitude is relatively small compared with VI $\left(S_{\mathrm{th}}\right)$ and VII $\left(S_{\mathrm{me}}\right)$. Considering the sink term VI $\left(S_{\mathrm{th}}\right)$, it is significant and its magnitude in Case B is bigger than those in Cases $\mathrm{G}$ and $\mathrm{H}$, since longer droplet residence time and more droplets in the computational domain lead to better heat exchange between the two phases. Finally, the mechanical work term VII $\left(S_{\mathrm{me}}\right)$ is the dominant source of FRIE through the "friction produces heat" principle. This effect due to the inter-phase drag has seldom been discussed, as many previous fundamental studies were conducted in the convection-free, counterflow diffusion flame configuration $[13,30]$. Moreover, the inter-phase drag is insignificant for fine water mist droplets $[13,30]$ compared with water spray droplets investigated in this study. As Fig. 5 shows, the magnitude of this term is directly proportional to the droplet momentum and consequently the inter-phase drag.

In terms of the magnitude, the heat release rate term III $\left(Q_{\mathrm{h}} \overline{\omega_{\mathrm{T}}}\right)$ is orders of magnitude larger than dropletrelated terms VI-IX, as seen in Fig. 5. This, however, does not suggest that the droplet-related terms are unimportant, as the (peak) gas temperature and reaction rate are severely suppressed in all droplet cases compared with Case A. It is important to note that turbulent flow, combustion and droplets interact in a highly nonlinear and dynamic way, and droplet-related terms also affect flow, turbulent kinetic energy, mixing, micromixing. The corresponding budget analysis, if possible, must be included to show the full physics involved. However, the overall effects of droplets on reaction rate are clearly demonstrated in Fig. 5. The pressure dilatation term II for the same cases has similar profiles, smaller magnitudes and opposite signs as compared with term III $\left(Q_{\mathrm{h}} \overline{\omega_{\mathrm{T}}}\right)$. The grid scale dissipation IV and subgrid scale dissipation V are both sink terms and are orders of magnitude smaller than the pressure-dilatation II and combustion released heat III. For brevity, these terms are not shown here.

\section{CONCLUSIONS}

A fundamental study of dynamic interactions between a buoyant reacting plume and evaporating droplets has been carried out using LES. An idealised prototype configuration is set up to numerically mimic a sprinkler nozzle placed right above the source of a small-scale fire, dispensing evaporating droplets of various properties. The gas phase of the reacting plume is described in the Eulerian frame while the discrete droplet phase is treated in the Lagrangian frame, with strong two-way coupling between the two phases through mass, momentum and energy exchange. Finite-rate chemistry is included through the use of an Arrhenius rate expression. The LES has incorporated a dynamic procedure to determine six model constants in the momentum, energy and species equations. The only empirically pre-set model constant is in the scale similarity subgrid-scale model for the reaction rate. A parametric study has been conducted by varying the initial Stokes number $\left(S t_{0}\right)$ or non-dimensional droplet size, mass loading ratio $\left(M L R_{0}\right)$ and droplet velocity magnitude $\left(\left|\mathbf{v}_{\mathrm{d} 0}\right|\right)$.

Droplets of three initial sizes $(780 \mu \mathrm{m}, 390 \mu \mathrm{m}$ and $195 \mu \mathrm{m})$ have distinctively different dynamic interactions with the reacting plume. Compared to the smallest mist droplets, the largest spray droplets can penetrate more easily the whole plume and have maximum suppression effects on the reaction. Detailed field analysis has been conducted to examine the complex nonlinear interactions among spray droplet dynamics, evaporation, turbulence and chemical reaction under different levels of $M L R_{0}$ (i.e., 0, 3, 6 and 9). Increasing $M L R_{0}$ progressively increases the droplet effects, and in the case of the largest droplets used, the reaction is completely suppressed and the plume structure destroyed. Increasing $M L R_{0}$ is more effective in causing flow reversal in the central plume region than increasing droplet velocity $\left|\mathbf{v}_{\mathrm{do}}\right|$ for the same initial droplet momentum. However, when $S t_{0}$ and $M L R_{0}$ are fixed, increasing $\left|\mathbf{v}_{\mathrm{d} 0}\right|$ has mixed effects. On the one hand, larger $\left|\mathbf{v}_{\mathrm{d} 0}\right|$ leads to higher droplet penetration capacity and lower plume upward velocity. On the other hand, it results in less droplet residence time in the computational domain and consequently shorter time for thermal cooling of the reacting plume. 


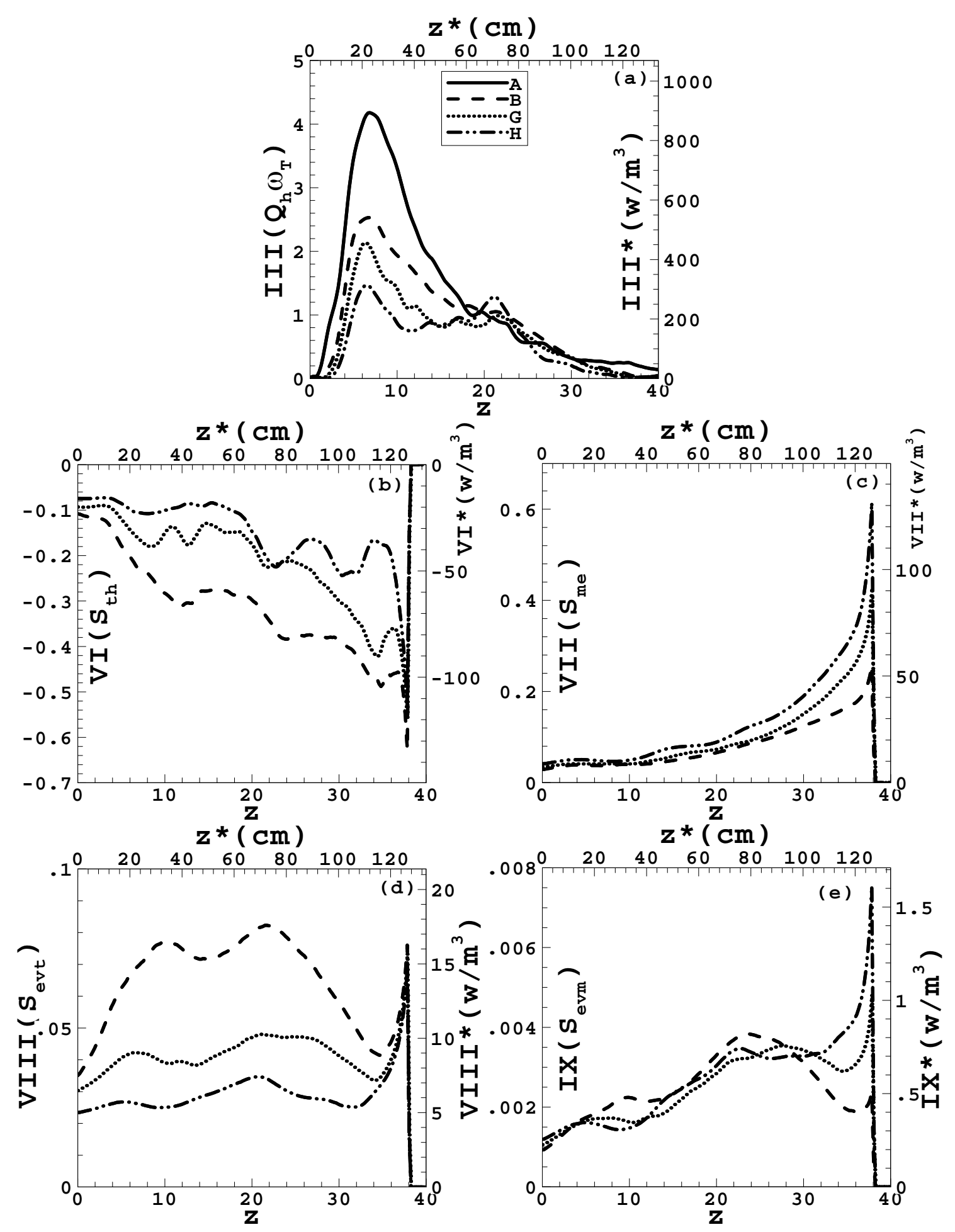

Fig. 5. FRIE budgets along the centreline: (a) the combustion released heat III $\left(Q_{\mathrm{h}} \overline{\omega_{\mathrm{T}}}\right)$ and droplet contributions: (b) VI $\left(S_{\mathrm{th}}\right)$, (c) VII $\left(S_{\mathrm{me}}\right)$, (d) VIII $\left(S_{\mathrm{evt}}\right)$ and (e) IX $\left(S_{\text {evm }}\right)$ in Eq. 1 for the Filtered Reduced Internal Energy (FRIE) of the reacting flow.

Finally, the budget equation for a non-dimensional gas temperature called the Filtered Reduced Internal Energy (FRIE) has been analyzed, with a focus on the droplet-related terms. One remarkable finding is that 
the only "cooling" effect on gas temperature comes from the convective heat transfer between the phases while there are three mechanisms (mechanical work done by droplet drag force, internal energy transfer into the gas phase due to evaporation and kinetic energy interactions between the phases) contributing to "warming" effects. On a purely order of magnitude analysis, all four droplet-related terms are very small compared with the heat release term. However, their importance in combustion suppression is amply demonstrated in the resulting significant reduction in reaction rate and gas temperature especially the peak values.

\section{ACKNOWLEDGEMENTS}

Funding from the EPSRC grant No. EP/E011640/1 and the BRE Trust are gratefully acknowledged. Supercomputing resources on the HPCx are from the UK Consortium on Computational Combustion for Engineering Applications (COCCFEA) under the EPSRC grant No. EP/D080223/1.

\section{REFERENCES}

[1] Grant, G., Brenton, J., and Drysdale, D., (2000) Fire Suppression by Water Sprays, Progress in Energy and Combustion Science 6: 79-130, doi:10.1016/S0360-1285(99)00012-X

[2] McCaffrey, B.J., (1984) Jet Diffusion Flame Suppression Using Water Sprays - An Interim Report, Combustion Science and Technology 40: 107-136, doi:10.1080/00102208408923801

[3] McCaffrey, B.J., (1989) Momentum Diffusion Flame Characteristics and the Effects of Water Spray, Combustion Science and Technology 63: 315-335, doi:10.1080/00102208908947134

[4] Hamins, A., and Mcgrattan, K.B., 2003. Reduced-Scale Experiments on the Water Suppression of a Rack-Storage Commodity Fire for Calibration of a CFD Fire Model. Fire Safety Science 7: 457468. doi:10.3801/IAFSS.FSS.7-457

[5] Nam, S., 2003. Application of Water Sprays to Industrial Oil Cooker Fire. Fire Safety Science 7: 469-480. doi:10.3801/IAFSS.FSS.7-469

[6] Garo, J.P., Richard, J., Souil, J.M., and Vantelon, J.P., 2003. On the Determination of Soot and Droplet Concentration and Velocity Fields during the Addition of a Water Mist on a Liquid Pool Fire at Laboratory Scale. Fire Safety Science 7: 507-518. doi:10.3801/IAFSS.FSS.7-507

[7] Carpenter, D., Kim, A.K., and Liu, Z., 2005. Application of Water Mist to Extinguish Large Oil Pool Fires for Industrial Oil Cooker Protection. Fire Safety Science 8: 741-752. doi:10.3801/IAFSS.FSS.8-741

[8] Schwille, J.A., and Lueptow, R.M., (2006) The Reaction of a Fire Plume to a Droplet Spray, Fire Safety Journal 41: 390-398, doi:10.1016/j.firesaf.2006.02.005

[9] Schwille, J.A., and Lueptow, R.M., (2006) Effect of a Fire Plume on Suppression Spray Droplet Motion, Atomization and Sprays 16: 563-577, doi:10.1615/AtomizSpr.v16.i5.60

[10] Fisher, B.T., Awtry, A.R., Sheinson, R.S., and Fleming, J.W., (2007) Flow Behavior Impact on the Suppression Effectiveness of Sub-10- $\mu \mathrm{m}$ Water Drops in Propane/Air Co-Flow Non-Premixed Flames, Proceedings of the Combustion Institute 31: 2731-2739, doi:10.1016/j.proci.2006.07.023

[11] Prasad, K., Li, C.P., and Kailasanath, K., (1999) Simulation of Water Mist Suppression of Small Scale Methanol Liquid Pool Fires, Fire Safety Journal 33: 185-212, doi:10.1016/S03797112(99)00028-4

[12] Prasad, K., Li, C., Kailasanath, K., Ndubizu, C., Ananth, R., and Tatem, P.A., (1998) Numerical Modeling of Water Mist Suppression of Methane-Air Diffusion Flames, Combustion Science and Technology 132: 325-364, doi:10.1080/00102209808952019

[13] Lentati, A.M., and Chelliah, H.K., (1998) Dynamics of Water Droplets in a Counterflow Field and their Effect on Flame Extinction, Combustion and Flame 115: 158-179, doi:10.1016/S0010$\underline{2180(97) 00355-6}$ 
[14] Kumar, S., Heywood, G.M., and Liew, S.K., 1997. Superdrop Modelling of a Sprinkler Spray in a Two-Phase CFD Particle-Tracking Model. Fire Safety Science 5: 889-900. doi:10.3801/IAFSS.FSS.5-889

[15] Holborn, P.G., Kumar, S., and Nolan, P.F., 2000. A 'Meandrop' Method for the Characterisation of a Sprinkler Spray in a Two-Phase CFD-Particle Tracking Model - An Improved Alternative to the 'Superdrop' Method. Fire Safety Science 6: 433-444. doi:10.3801/IAFSS.FSS.6-433

[16] Nam, S., (1996) Development of a Computational Model Simulating the Interaction between a Fire Plume and a Sprinkler Spray, Fire Safety Journal 26: 1-33, doi:10.1016/0379-7112(96)00015$\underline{X}$

[17] Nam, S., (1999) Numerical Simulation of the Penetration Capability of Sprinkler Sprays, Fire Safety Journal 32: 307-329, doi:10.1016/S0379-7112(99)00004-1

[18] Yoon, S.S., Kim, H.Y., DesJardin, P.E., Hewson, J.C., Tieszen, S.R., and Blanchat, T.K. (2007) Unsteady RANS Modeling of Water-Spray Suppression for Large-Scale Compartment Pool Fires, Atomization and Sprays 17: 1-45, doi:10.1615/AtomizSpr.v17.i1.10

[19] Luo, K.H., "New Opportunities and Challenges in Fire Dynamics Modelling," Proceedings of the Fourth International Seminar on Fire and Explosion Hazards, 2004, pp. 39-52.

[20] Xia, J., Luo, K.H., and Kumar, S., (2008) Large-Eddy Simulation of Interactions Between a Reacting Jet and Evaporating Droplets, Flow Turbulence and Combustion 80: 133-153, doi:10.1007/s10494-007-9084-4

[21] Spalding, D.B., (1953) The Combustion of Liquid Fuels, Proceedings of the Combustion Institute 4: 847-864.

[22] Luo, K.H., (1999) Combustion Effects on Turbulence in a Partially Premixed Supersonic Diffusion Flame, Combustion and Flame 119: 417-435, doi:10.1016/S0010-2180(99)00074-7

[23] Fernández-Tarrazo, E., Sánchez, A.L., Liñán, A., Williams, F.A., (2006) A Simple One-Step Chemistry Model for Partially Premixed Hydrocarbon Combustion, Combustion and Flame 147: 32-38, doi:10.1016/j.combustflame.2006.08.001

[24] DesJardin, P.E., and Frankel, S.H., (1998) Large Eddy Simulation of a Nonpremixed Reacting Jet: Application and Assessment of Subgrid-Scale Combustion Models, Physics of Fluids 10: 22982314, doi:10.1063/1.869749

[25] Poinsot, T.J., and Lele, S.K., (1992) Boundary-Conditions for Direct Simulations of Compressible Viscous Flows, Journal of Computational Physics 101: 104-129, doi:10.1016/00219991(92)90046-2

[26] Thompson, K.W., (1987) Time-Dependent Boundary-Conditions for Hyperbolic Systems, Journal of Computational Physics 68: 1-24, doi:10.1016/0021-9991(87)90041-6

[27] Sandhu, H.S. and Sandham, N.D., "Boundary Conditions for Spatially Growing Compressible Shear Layers," Queen Mary and Westfield College Report QMW-EP-1100, London, UK, 1994, 24 p.

[28] Germano, M., Piomelli, U., Moin, P., and Cabot, W.H., (1991) A Dynamic Subgrid-Scale Eddy Viscosity Model, Physics of Fluids A 3: 1760-1765, doi:10.1063/1.857955

[29] Sheppard, D.T., and Lueptow, R.M., (2005) Characterization of Fire Sprinkler Sprays Using Particle Image Velocimetry, Atomization and Sprays 15: 341-362, doi:10.1615/AtomizSpr.v15.i3.50

[30] Dvorjetski, A., and Greenberg, J.B., (2004) Theoretical Analysis of Polydisperse Water Spray Extinction of Opposed Flow Diffusion Flames, Fire Safety Journal 39: 309-326, doi:10.1016/j.firesaf.2003.11.009 